\title{
Label-Free Immunoassay of Carcinoembryonic Antigen by Microfluidic Channel Biosensor Based on Imaging Ellipsometry and Its Clinic Application
}

\section{Chenghong Huang, Baixue Wang, Yanyan Chen \& Gang Jin}

To cite this article: Chenghong Huang, Baixue Wang, Yanyan Chen \& Gang Jin (2020) LabelFree Immunoassay of Carcinoembryonic Antigen by Microfluidic Channel Biosensor Based on Imaging Ellipsometry and Its Clinic Application, Integrated Ferroelectrics, 209:1, 19-29, DOI: 10.1080/10584587.2020.1728800

To link to this article: https://doi.org/10.1080/10584587.2020.1728800

曲 Published online: 01 Jul 2020.

Submit your article to this journal ¿

山 Article views: 8

View related articles $₫$

View Crossmark data $₫$ 


\title{
Label-Free Immunoassay of Carcinoembryonic Antigen by Microfluidic Channel Biosensor Based on Imaging Ellipsometry and Its Clinic Application
}

\author{
Chenghong Huang ${ }^{a}$, Baixue Wang ${ }^{b}$, Yanyan Chen ${ }^{c}$, and Gang Jin ${ }^{d}$ \\ ${ }^{a}$ Chongqing Key Laboratory of Industrial Fermentation Microorganism, Chongqing University of Science

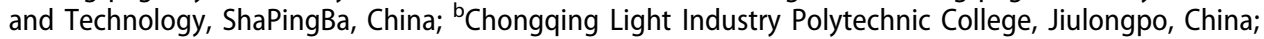 \\ 'Suzhou Institute of Nano-Tech and Nano-Bionics, Chinese Academy of Science, Beijing, China; \\ ${ }^{d}$ Institute of Mechanics, Chinese Academy of Science, Beijing, China
}

\begin{abstract}
This study represents a label-free immunoassay for carcinoembryonic antigen (CEA) by microfluidic biosensor based on imaging ellipsometry (BIE) and its application is further validated by clinic sera. The sandwich format of analysis configuration was adopted, in which Rat-mAb was served as capture antibody, rabbit anti-CEA polyclonal antibody (rabbit-pAb) as detection antibody and goat anti-rabbit IgG (goat-lgG) as an enhancer added to increase detection signal. The methodology includes rat-mAb concentration screening, rabbit-pAb and goat-IgG concentration optimization, creation of dose-response curve and determination of cutoff value. Results show the linear range is from $4.0-64.0 \mathrm{ng} / \mathrm{ml}$ with a lower limit of $1.0 \mathrm{ng} / \mathrm{ml}(\mathrm{S} / \mathrm{N}=3)$. Application of the BIE to clinic samples demonstrated that the relation coefficient is 0.94 and substantial agreement with the results of ECLIA's (Kappa $=0.733, U=4.35>\mathrm{U}_{0.01}$ ). The proposed method exhibits the potential for application.
\end{abstract}

\section{ARTICLE HISTORY}

Received 16 September 2019 Accepted 3 February 2020

\section{KEYWORDS}

Imaging ellipsometry; carcinoembryonic antigen; immunoassay

\section{Introduction}

Carcinoembryonic antigen (CEA), a glycoprotein of approximately $180 \mathrm{KDa}$ molecular weight [1], is often served as tumor maker relating to colon carcinomas [2], breast cancer [3], pancreatic cancer [4], lung cancer [5], and gynecologic malignancies [6]. Its highly sensitive detection and accurate analysis in serum is very important for cancer diagnosis and management. To date, various immunoassays, such as radioimmune immunoassay [7], Enzyme linked immunosorbent assay (ELISA) [8] and chemiluminescence immunoassay (ECLIA) [9], have been developed for CEA detection. Although possessing high sensitivity and excellent specificity, in many cases, these methods could still not meet the requirements of rapid and high throughput detection when large quantity samples in hospital to be analyzed. Furthermore, some of them might suffer from troubles of labeling annoyance by enzyme [10], radioactive substances, fluorescence tags [11], or metal nano-particles [12]. Contrary to above mentioned techniques, label-free method offers advantages as a direct means of detecting protein-protein 
binding. Surface plasmon resonance (SPR), electrochemical-impedance (EI), atomic force microscopy (AFM) and quartz crystal microbalance (QCM) have ever been reported to be suitable non-labeling detection methods. Although SPR technique matured to become a versatile tool, it is usually troubled by fabricating a glass based metal substrate due to an interface for generation of surface plasmon is required [13]. Electrochemical-impedance is difficult to realize high throughput. AFM manipulation has the disadvantages of destructive to sample and specific work environment. The major drawback of QCM is that the manipulation is often operated in dry air or nitrogen in order to guarantee identical conditions before and after each measurement of the process [14]. Thus, it is difficult to obtain an immunoassay with advantages of label-free, good sensitivity and easy operation.

Biosensor based on imaging ellipsometry (BIE), a label-free and nondestructive characterization technique for thin layer $[15,16]$, has greatly progressed since the concept put forward in 1995 [17]. It is a direct optical visualization method that offers a distinct graph for qualitative or quantitative analysis. Compared with above-mentioned techniques, BIE has advantages of rapid, high sensitivity and simplicity [18-20]. BIE has already been successfully applied in the field of monitoring antigen-antibody kinetics [21], bacteria examination [22] and virus detection [23, 24].

Here, we describe a label-free immunoassay for CEA detection utilizing BIE system with sensitivity of $1.0 \mathrm{ng} / \mathrm{ml}$. The assay strategy is that anti-CEA monoclonal antibody (rat-mAb) is firstly immobilized on modified silicon wafer, after CEA captured, rabbit anti-CEA polyclonal antibody (rabbit-pAb) was added to combine with the captured CEA and goat anti-rabbit IgG (goat-IgG) as an enhancer subsequently delivered to combine with the bounded rabbit-pAb. The combined protein amount can be measured quantitatively by imaging ellipsometry. The ensemble of methods including rat-mAb concentration screening, rabbit-pAb and goat-IgG concentration optimization, antigen reaction time are described. In clinic application perspective, cutoff value for colon cancer diagnosis is determined and diagnosis value is also validated.

\section{Materials and methods}

\subsection{Reagents}

Silicon wafers were purchased from General Research Institute for Nonferrous Metals (China). Human carcinoembryonic antigen (CEA), Rat anti-human CEA monoclonal antibody (rat-mAb), rabbit anti-human CEA polyclonal antibody (rabbit-pAb) and goat anti-rabbit IgG (goat IgG) were purchased from KangWei Co. Ltd. (China). 3aminopropyltriethoxysilane (APTES) and Glutaraldehyde (GA) were from Sigma. Fetal bovine serum (FBS) was from DingGuo Co.Ltd (in China). Other used chemicals were analytical grade or better. Water was obtained from a millipore Milli-Q ion exchange apparatus. Phosphate-buffered saline (PBS, pH 7.4) was prepared in deionized water.

\subsection{Bie system}

BIE system consists of microfluidic array system and imaging ellipsometry (IE). The microfluidic array system is used for surface patterning and array fabrication. IE is used for reading the protein array. A polydimethylsiloxane (PDMS) template in microfluidic 
array system contains an $8 \times 6$ cell array. When silicon wafer was attached onto the template, 48 individual chambers are formed independently. Each chamber has two access holes, namely, an inlet and an outlet, for solution passage. Solution can pass in and out of the chamber for oriented delivery through Teflon tubes connection. Protein solutions are delivered individually to different cells under the negative pressure generated by micropump (24 channel, ISMATEC, Switzerland. www.ismatec.com) so that the surface is patterned homogeneously and simultaneously in array format [25]. Then, 48 protein dots were patterned regularly on silicon wafer surface.

Imaging ellipsometry was used for the visualization and quantification of the protein adsorption layer on the surface of silicon substrate. It is a powerful tool for optical characterization of thin film and very sensitive to significant change in optical parameter with a sub-nanometer resolution of vertical [26]. During experiments, the optical components in the system are adjusted to fulfill the null conditions on a silicon wafer without any adsorbed protein layers and to measure the adsorption layer thickness when the protein was adsorbed. Under this condition, the detected signal density $I$ is related to the thickness $(d)$ of the protein layer according to $I=k d^{2}$, where $k$ is a constant [27]. Meanwhile, there is a relationship between surface concentration and film thickness: surface concentration $\left(\mu \mathrm{g} / \mathrm{cm}^{2}\right) \approx \mathrm{k} \times d(\mathrm{~nm})$, where $k=0.12$. The ellipsometry image was focused on the protein area of the CCD camera. A digital image was grabbed by and stored in a computer with a grayscale format ( 8 bits, 0-255 grayscale) for further evaluation by image-processing software of the program.

\subsection{Anti-CEA monoclonal antibody immobilization}

Silicon wafers were firstly rinsed thoroughly by deionized water. Then, silicon wafers were made hydrophilic by immersion into the mixture solution of $\mathrm{H}_{2} \mathrm{O}_{2} / \mathrm{H}_{2} \mathrm{SO}_{4}$ (1:3, v/ v) for $30 \mathrm{~min}$. The oxidized silicon wafers were subsequently incubated in a mixture of 3-aminopropyltriethoxysilane (APTES) and ethanol solution $(1: 15, \mathrm{v} / \mathrm{v})$ for $2 \mathrm{~h}$. This step makes the silicon wafers carrying a layer of $-\mathrm{NH}_{2}$ densely. Next, the silicon wafers were placed in a solution of glutaraldehyde (GA) in PBS (1:10, v/v) for $1.5 \mathrm{~h}$ [28] incubation. The prepared silicon wafers were at last stored in $4^{\circ}$ Crefrigrator for use.

Rat-mAb immobilization was carried out using 48-channel poly(dimethylsiloxane) (PDMS) patterning template prepared as described previously [25]. $20 \mu \mathrm{l}$ rat-mAb was deposited onto the modified silicon wafer by microfluidic channels for 20 min incubation. Each channel was subsequently rinsed by $100 \mu \mathrm{l}$ PBST (PBS containing 0.05\% Tween-20) for $10 \mathrm{~min}$. For decrease nonspecific adsorption, all channels were blocked by bovine fetal serum (FBS, $1: 10$ ) at $1.0 \mu \mathrm{l} / \mathrm{min}$ for $30 \mathrm{~min}$. Then, the capturing layer for CEA detection was achieved.

\subsection{CEA detection}

The procedure in general for CEA detection includes the following steps: first, $20 \mu \mathrm{l}$ CEA reference or sample was dispensed to the channel at $1.0 \mu \mathrm{L} / \mathrm{min}$ for $20 \mathrm{~min}$. The channel was then rinsed by PBST at $20 \mu \mathrm{l} / \mathrm{min}$ for $5 \mathrm{~min}$. Later, rabbit-pAb was added to combine with the captured antigen at $1.0 \mu \mathrm{L} / \mathrm{min}$ for $15 \mathrm{~min}$. After washed by PBST 
at $20.0 \mu \mathrm{l} / \mathrm{min}$ for $5 \mathrm{~min}$ again, goat IgG was delivered to combine with the rabbit-pAb at $1.0 \mu \mathrm{l} / \mathrm{min}$ for $15 \mathrm{~min}$. Finally, the silicon wafer was taken out of the template and rinsed intensely by deionized water and dried under a stream of nitrogen. The total assay time for CEA was approximately $60 \mathrm{~min}$.

\subsection{Serum application}

Sera were aliquoted and stored at $-75^{\circ} \mathrm{C}$ until the time of analysis. For sample test, the analysis was strictly complied with the procedure established. Each sample was tested twice and the mean was calculated. Meanwhile, the sera were measured by ECLIA according to the specification.

\subsection{Statistical analysis}

Parametric results of healthy and patient group were expressed as mean \pm standard deviation (SD) and difference between groups was compared by independent-samples Test. Results comparison between BIE's and ECLIA's made using bivariate Spearman's correlation test (A $P$ value $<0.05$ was considered as statistically significant). Agreement was performed by kappa analysis.

\section{Results and discussions}

\subsection{Optimization of analytical conditions}

Serial diluted rat-mAb $(40.0,80.0,100.0,120.0,140.0$ and $160.0 \mu \mathrm{g} / \mathrm{ml})$ were dispensed to the glutaraldehyde surface. We observed that the saturation was gradually arrived at $140.0 \mu \mathrm{g} / \mathrm{ml}$ upward. Subsequently, these immobilized antibodies were separately combined with CEA with the same concentrations of $4.0 \mathrm{ng} / \mathrm{ml}$. The maximum grayscale change was served as the criterion to evaluate the best suitable concentration. It was found that $120.0 \mathrm{ug} / \mathrm{ml}$ has optimum antigen binding capacity.

Concentration of rabbit-pAb and goat-IgG were systematically optimized. Rat-mAb was firstly immobilized on aldehyde surface, then, CEA with 4.0, 8.0, 16.0, 32.0 and $64.0 \mathrm{ng} / \mathrm{ml}$ were added to combine with immobilized rat-mAb. Later, rabbit-pAb of 1:5, $1: 10,1: 15,1: 30$ and 1:50 were added to combine with captured CEA and goat-IgG of $1: 10,1: 20,1: 30$ and 1:40 were delivered to combine with rabbit-pAb for optimization of optimal dose response and best detection limit. There was a significant difference in efficacy of different concentrations of rabbit-pAb and goat-IgG for grayscale response. For rabbit-pAb, dilution rate $<1: 15$ results in a narrow dynamic range. Dilution ratio exceeding 1:15 generally leads to disturbance with the assay results as its high viscosity in microfluidic system, which also previously noted by other researchers. When goat IgG was introduced, 1:20 dilution ratio generates grayscale response with a desirable detection range and a preferable detection limit. Since the output signals from CCD camera were limited to the range $0-255$, we select $1: 15$ of rabbit-pAb and 1:20 of goatIgG as work concentration.

The influence of the reaction time on grayscale response was also investigated. When the antigens reach the capturing layer or rabbit-pAb and goat-IgG reach the antigens sites, 
it takes time to form antigen-antibody complex. The incubation time was 5, 10, 15, 20, 25 and $30 \mathrm{~min}$, using the same CEA concentration $(100.0 \mathrm{ng} / \mathrm{ml})$ to evaluate the effect. The grayscale response observed with the incubation time up to $20 \mathrm{~min}$ and after that the reactions steadily reach to equilibrium. Therefore, the incubation time of $20 \mathrm{~min}$ was adopted.

\subsection{Calibration curve and sensitivity}

Dose-response of CEA was obtained according to optimized reaction conditions. Figure 1 illustrates a representative ellipsometric platform (1A) and analysis system(1B). Figure 2 represent a typical protein square dot after sample application. It shows that protein dots turn brighter with raised CEA concentration (Figure 2A). Dot 1 was served as antibody control and dot 2 as blank control(BSA blocking). The CEA concentrations from dot3-12 were $0.0,1.0,2.0,4.0,8.0,16.0,32.0,64.0,128.0,256.0 \mathrm{ng} / \mathrm{ml}$, respectively. The grayscale image can be transferred into three-dimension distribution for intuitionistic observation (Figure 2B). The tests were performed in three independent tests, and the mean grayscale value was used for a nonlinear regression analysis. By 4-parameter logistic algorithms, a dose-response curve was obtained in Figure 3. The curve can be represented by the regression equation: $\mathrm{Y}=-29.3+93.6 \log \mathrm{X}\left(\mathrm{R}^{2}=0.989, \mathrm{P}<0.01\right)$,

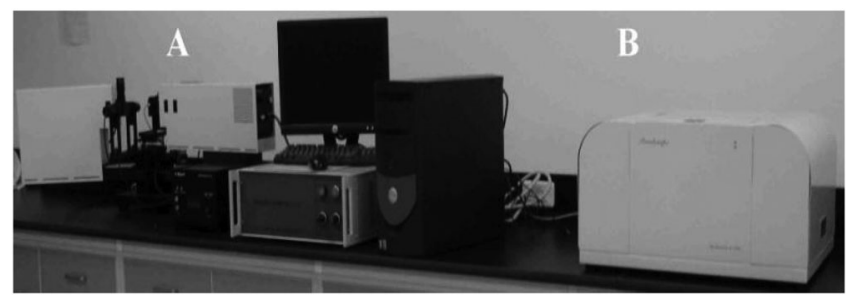

Figure 1. The illustration of Bie system. (A) imaging ellipsometry system and (B) array fabrication and microfluidic system.

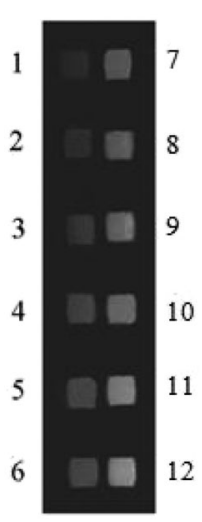

(A)

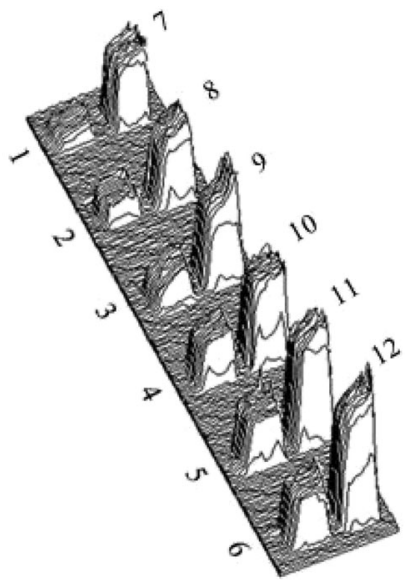

(B)

Figure 2. Typical dose-response ellipsometric image (A) and corresponding three-dimension distribution map (B). dots 1 and 2 were served as antibody and blocking control, respectively. Dots from 3-12 were CEA with $0.0,1.0,2.0,4.0,8.0,16.0,32.0,64.0,128.0$, and $256.0 \mathrm{ng} / \mathrm{ml}$, respectively. 


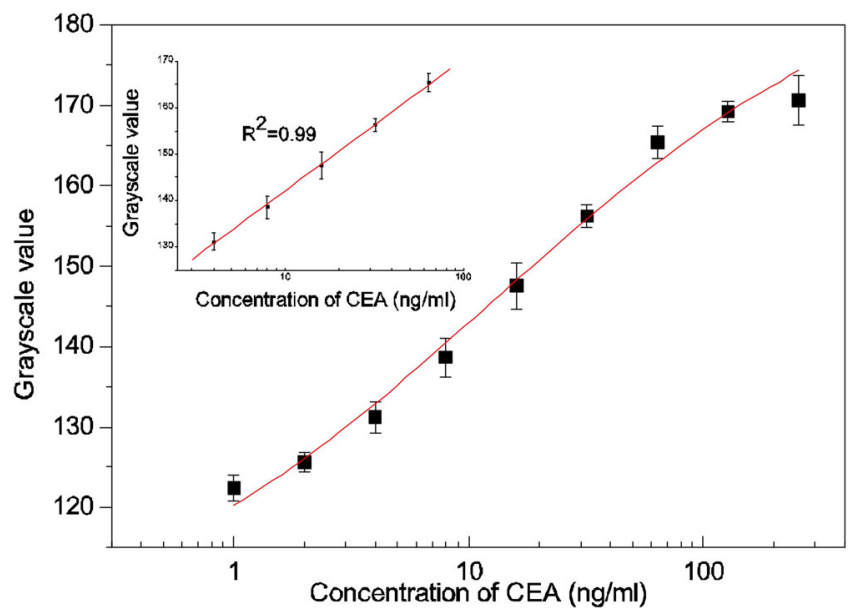

Figure 3. Calibration curve of the grayscale values vs. CEA concentration obtained under optimized assay conditions. Data were three independent tests and the mean value was calculated. Inset shows linear calibration curve.

where $\mathrm{Y}$ denotes the grayscale value and $\mathrm{X}$ is the logarithmic transformation of CEA concentration $(\mathrm{ng} / \mathrm{ml})$. The minimum detection limit can be as low as $1.0 \mathrm{ng} / \mathrm{ml}$ $(\mathrm{S} / \mathrm{N}=3)$. The linear range was $4.0-64.0 \mathrm{ng} / \mathrm{ml}$ (Inset).

\subsection{Reproducibility, accuracy, selectivity and stability}

As there were no existing validation guideline for biomarkers and protein array, the reproducibility of the immunoassay was investigated using the Food and Drug Administration (FDA) regulatory guidelines for pharmacokinetic assay validation [29]. The reproducibility was assessed by $\mathrm{CV} \%$, defined as $\mathrm{CV} \%=\sigma / \bar{x} \times 100 \%$, where $\sigma$ is standard deviation and $\bar{x}$ is mean grayscale value. The assessments were conducted by three quality control sera with low $(8.2 \mathrm{ng} / \mathrm{ml})$, middle $(30.6 \mathrm{ng} / \mathrm{ml})$ and high $(58.7 \mathrm{ng} /$ $\mathrm{ml}$ ) CEA concentrations. The intra-slide and the inter-slide reproducibility were $6.1 \%$, $8.2 \%, 9.1 \%$ and $14.2 \%, 9.8 \%$ and $13.4 \%$, respectively.

Accuracy was appraised by spike recovery test that a known amount of analyte was added (spiked) into the natural test sample matrix and its response was measured (recovered) in the assay by comparison to an identical spike in the standard. We employed a sample without CEA to perform the test by adding 0.0, 10.0, 20.030 .0 and $40.0 \mathrm{ng}$ for recovery rate calculation. The range was from $94.9 \%$ to $107.0 \%$.

Selectivity was evaluated by three similar tumor markers including alpha-fetoprotein (AFP), carbohydrate antigen 199(CA199), carbohydrate antigen 242 (CA 242) and a common protein human serum albumin (HSA) in this study. The results were shown in Figure 4 . The cross reaction rates of all tested substances were no more than $5.0 \%$ compared with $100 \mathrm{ng} / \mathrm{ml}$ CEA.

The stability of the prepared silicon slide was tested over a week period, when they were stored in the refrigerator at $4{ }^{\circ} \mathrm{C}$, it retains $94 \%$ of its initial ability for antibody loading and antigen binding capacity. 


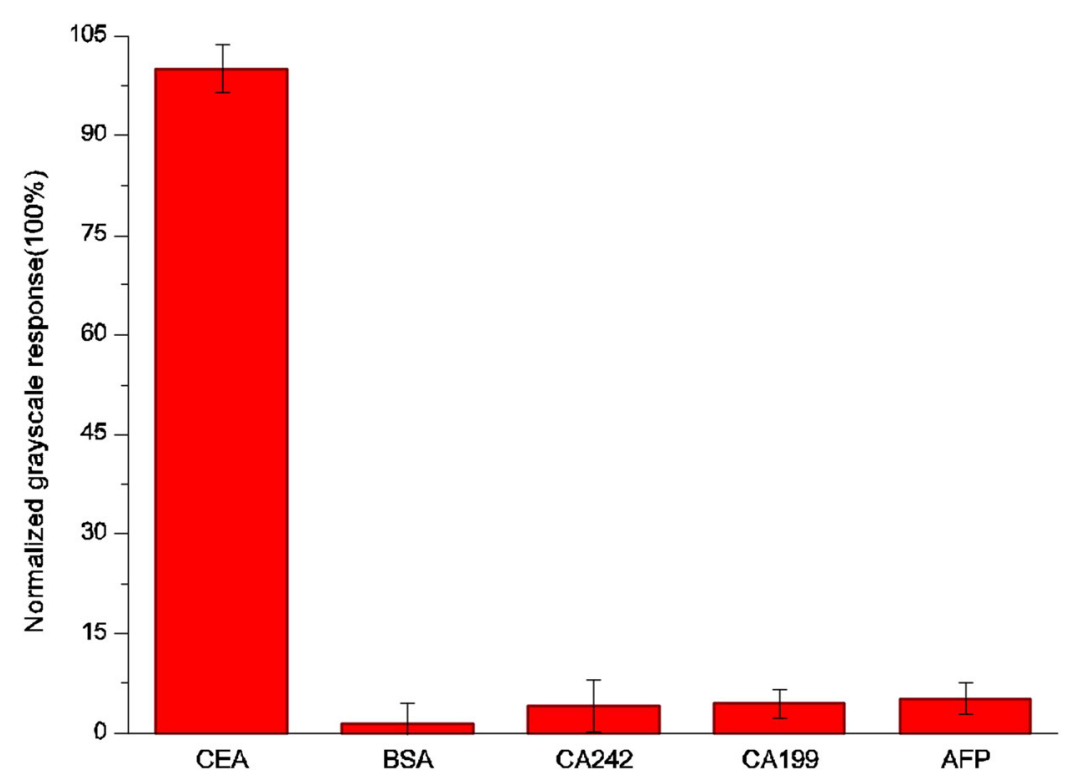

Figure 4. Selectivity of Bie evaluated by CEA $(32 \mathrm{ng} / \mathrm{ml})$, BSA $(1 \mathrm{ug} / \mathrm{ml})$, CA242 $(100 \mathrm{ng} / \mathrm{ml})$, CA199 $(100 \mathrm{ng} / \mathrm{ml})$ and AFP $(100 \mathrm{ng} / \mathrm{ml})$.

\subsection{Cutoff value determination}

As we all known, sensitivity and specificity, which are defined as the number of true positive decisions/the number of actually positive cases and the number of true negative decisions/the number of actually negative cases, respectively, constitute the basic measures of performance of diagnostic tests [30]. Different cutoff levels produce multiple pairs of sensitivity and specificity values. One can draw a graph using the sensitivities as the $\mathrm{y}$ coordinates and the 1-specificities as the $\mathrm{x}$ coordinates. A receiver operating characteristic curve (ROC) can be generated and the area under ROC can be used for evaluation of diagnosis value. Herein, cutoff values were determined by referring to ROC with the help of pathology test as reference criterion. Discrete grayscale changes $(\Delta G)$ were selected as cutoff value ("threshold") to count true positive or false negative for 49 patient samples and true negative or false positive for 100 healthy samples. The true positive fraction, false positive fraction and Youden's index were then tabulated in Table 1. When the Youden's index reached maxima, the corresponding variation in grayscale value was taken as the best cutoff value [31]. The best cutoff point was determined 10.0, where the sensitivity is 0.57 and the specificity is 0.49 . After grayscale transferred into mass concentration, the corresponding value was $5.8 \mathrm{ng} / \mathrm{ml}$.

\subsection{Serum testing}

Total 49 sera samples from colon cancer patient were used for a preliminary study. We also collected 100 serum samples from healthy as negative control. The CEA distributions (by BIE) of the healthy group and the patient group are shown in Figure 5. There is a significance difference of grayscale change between healthy $(5.32 \pm 4.16)$ and patient group $(28.57 \pm 16.57)(F=108.24, P<0.01)$. After transferred into mass concentration, 
Table 1. Cutoff values for CEA detection. The variation in grayscale value for CEA detection is above then negative control. TPF is true positive/true positive fraction; FPF is false positive/false positive fraction; positive likelihood ratio is TPF divided by FPF (TPF/FPF); youden's index is TPF minus FPF. The cutoff value for disease diagnosis is indicated by the line with boldfaced italics.

\begin{tabular}{lllcc}
\hline$\triangle \mathrm{G}$ & TPF & FPF & Positive likelihood ratio & Youden's index \\
\hline 1 & 0.97 & 0.91 & 1.07 & 0.06 \\
2 & 0.93 & 0.75 & 1.24 & 0.18 \\
3 & 0.89 & 0.65 & 1.37 & 0.24 \\
4 & 0.85 & 0.55 & 1.55 & 0.30 \\
5 & 0.79 & 0.44 & 1.79 & 0.35 \\
6 & 0.76 & 0.35 & 2.17 & 0.41 \\
7 & 0.74 & 0.3 & 2.47 & 0.44 \\
8 & 0.72 & 0.22 & 3.27 & 0.50 \\
9 & 0.69 & 0.16 & 4.31 & 0.53 \\
10 & 0.67 & 0.11 & 6.09 & 0.56 \\
11 & 0.64 & 0.09 & 7.11 & 0.55 \\
12 & 0.60 & 0.09 & 6.67 & 0.51 \\
13 & 0.57 & 0.07 & 8.14 & 0.50 \\
14 & 0.57 & 0.06 & 9.50 & 0.51 \\
15 & 0.55 & 0.03 & 18.33 & 0.52 \\
16 & 0.54 & 0.02 & 27.00 & 0.52 \\
17 & 0.51 & 0.01 & 51.00 & 0.50 \\
22 & 0.46 & 0.00 & 1 & 0.46 \\
\hline
\end{tabular}

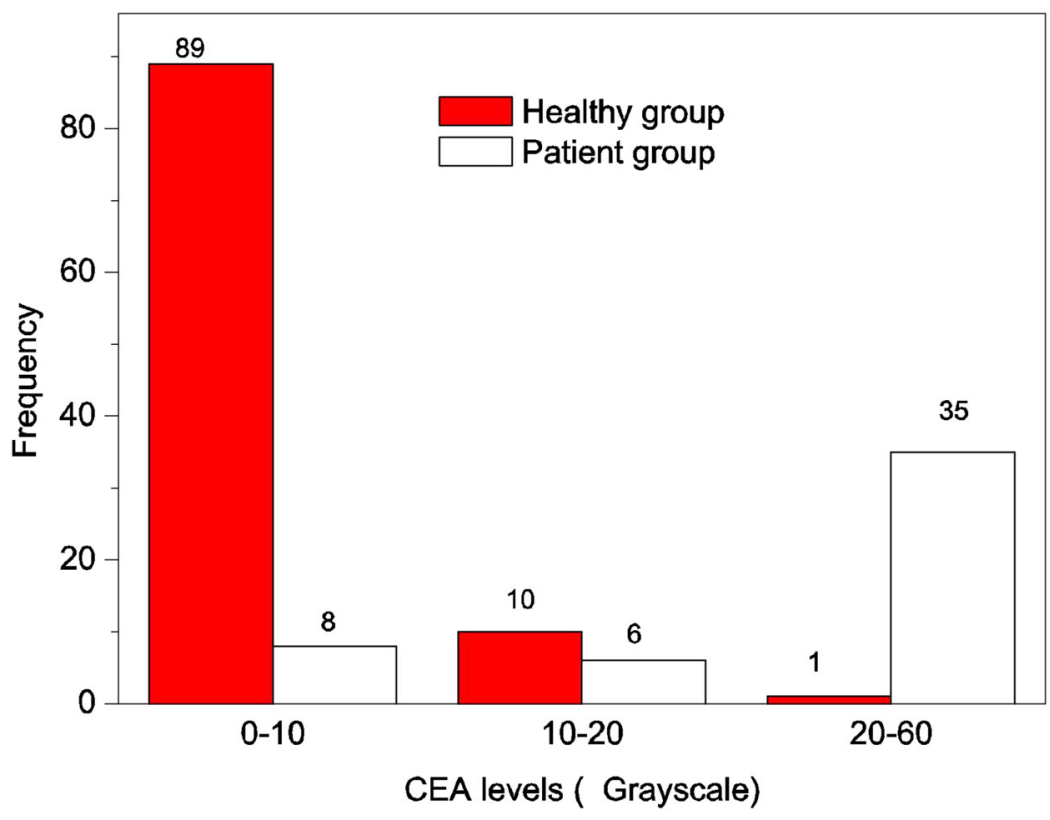

Figure 5. Grayscale changes for healthy group and patient group samples measured by Bie.

CEA in healthy group are mainly in the scope under $10 . \mathrm{ng} / \mathrm{ml}$ while in the scope $20.0 \mathrm{ng} / \mathrm{ml}$ upward in patient group. The positive rate for healthy and patient group are $10 \%(10 / 100)$ and $69 \%(34 / 49)$, respectively. Both positive rate and serum level of CEA in patient group were significantly higher than that in healthy group. The area under the ROC curve for differentiating people with cancer from healthy were 0.877 (95\% confidence interval, 0.69-0.919) for the BIE test and 0.892 (95\% confidence interval, 


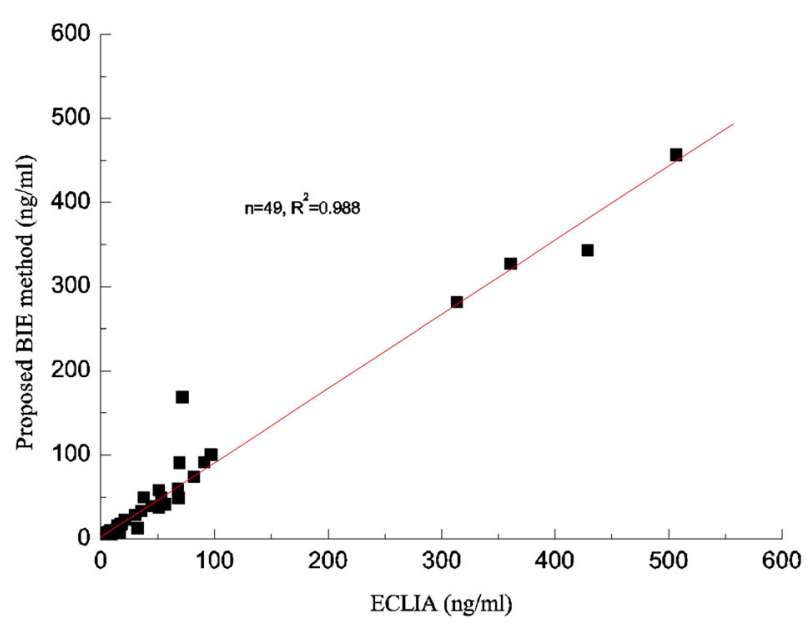

Figure 6. Correlation of CEA levels in sera between conventional ECLIA method and the Bie method.

0.776-0.998) for the ECLIA test. It signify this method hold the potential for largescale screening.

\subsection{Comparison with ECLIA}

The quantitative results by BIE against by ECLIA (which was often taken as "gold standard" in clinical application were plotted. As shown in Figure 6, the correlation can be described by the equation: $y=2.42+0.88 \mathrm{x},\left(n=49, \mathrm{R}^{2}=0.988, \mathrm{p}<0.01\right)$. Using the Kappa analysis to measure the agreement [32], there is a middle agreement for diagnosing colon cancer between two methods (Kappa $=0.535, U=2.729>\mathrm{U}_{0.01}$ ).

\section{Conclusion}

To summary, Rat-mAb was covalently immobilized on modified silicon substrate and a capturing layer was achieved after BSA blocking. Then, rabbit anti-CEA polyclonal antibody was added to combine with captured antigen and goat anti-rabbit IgG was added to combine with rabbit polyclonal antibody. Results can be measured directly by imaging ellipsometry. The proposed method has a detection limit of $1.0 \mathrm{ng} / \mathrm{mL}$. The detection time is approximately $60 \mathrm{~min}$. Preliminary application demonstrated that it has an area under ROC of 0.877 to distinguish between colon cancer and healthy sera. The proposed method has a substantial agreement with ECLIA's and the potential for largescale serum screening.

\section{Funding}

This work was supported by the Open Fund of Chongqing Key Laboratory of Industrial Fermentation Microorganism(LIFM201712) and NSF of Chongqing(cstc2016shmszx80080 and cstc2019jscx-msxm1542) and fund of Chongqing Education Board (KJ1605701). 


\section{References}

1. Johan. The chemistry of carcinoembryonic antigen, Cancer 36, 2445 (1975).

2. M. S. Kahlenberg et al., Molecular prognostics in colorectal cancer, Surg. Oncol. 12 (3), 173 (2003). DOI: 10.1016/S0960-7404(03)00006-9.

3. E. G.Mansour et al., Tissue and plasma carcinoembryonic antigen in early breast cancer a prognostic factor, Cancer 51 (7), 1243 (1983). DOI: 10.1002/1097-0142(19830401)51:7<1243::AIDCNCR2820510712>3.0.CO;2-A.

4. M. S. Sawhney et al. Comparison of carcinoembryonic antigen and molecular analysis in pancreatic cyst fluid, Gastrointest. Endosc. 69 (6), 1106 (2009). DOI: 10.1016/j.gie.2008.08.015.

5. S. S. Katsunari Matsuoka et al., Prognostic value of carcinoembryonic antigen and CYFRA21-1 in patients with pathological stage I non-small cell lung cancer, Eur. J. CardioThorac. 32 (3), 435 (2007). DOI: 10.1016/j.ejcts.2007.05.014.

6. J. R. v Nageil et al., The clinical significance of carcinoembryonic antigen in the plasma and tumors of patients with gynecologic malignancies, Cancer 42, 1527 (1978).

7. M. L.Egan, J. E. Coligan, and C. W. Todd, Radioimmune assay for the diagnosis of human cancer, Cancer 34 (S8), 1504 (1974). DOI: 10.1002/1097-0142(197410)34:8+<1504::AIDCNCR2820340823>3.0.CO;2-Q.

8. S. R. Urva, V. C. Yang, and J. P. Balthasar, Development and validation of an enzyme linked immunosorbent assay for the quantification of carcinoembryonic antigen in mouse plasma, J. Immunoass. Immunoch. 30 (4), 418 (2009). DOI: 10.1080/15321810903188227.

9. J. Lin, F. Yan, and H. Ju, Noncompetitive enzyme immunoassay for carcinoembryonic antigen by flow injection chemiluminescence, Clin. Chim. Acta 341 (1-2), 109 (2004). DOI: 10. 1016/j.cccn.2003.11.014.

10. J. Wu et al., A disposable electrochemical immunosensor for flow injection immunoassay of carcinoembryonic antigen, Biosens. Bioelectro. 22 (1), 102 (2006). DOI: 10.1016/j.bios. 2005.12.008.

11. F. Yan et al., Flow injection immunoassay for carcinoembryonic antigen combined with time-resolved fluorometric detection, J. Immunol. Methods 305 (2), 120 (2005). DOI: 10. 1016/j.jim.2005.03.004.

12. J. V. Jokerst et al., Nano-bio-chips for high performance multiplexed protein detection:Determinations of cancer biomarkers in serum and saliva using quantum dot bioconjugate labels, Biosens. Bioelectro. 24 (12), 3622 (2009). DOI: 10.1016/j.bios.2009.05.026.

13. Y. M. Bae et al., Detection of insulin-antibody binding on a solid surface using imaging ellipsometry, Biosens. Bioelectro. 20 (4), 895 (2004). DOI: 10.1016/j.bios.2004.03.032.

14. S. P. Sakti et al., Disposable HSA QCM-immunosensor for practical measurement in liquid, Sens. Actuat. B Chem. 78 (1-3), 257 (2001). DOI: 10.1016/S0925-4005(01)00822-X.

15. H. Arwin, Ellipsometry on thin organic layers of biological interest: Characterization and applications, Thin Solid Films 377-378, 48 (2000). DOI: 10.1016/S0040-6090(00)01385-7.

16. T. Schram et al., Spectroscopic ellipsometry: A non-destructive technique for surface analysis, Adv. Eng. Mater. 1 (1), 63 (1999). DOI: 10.1002/(SICI)1527-2648(199909)1:1<63::AIDADEM63>3.0.CO;2-1.

17. G. Jin et al., A biosensor concept based on imaging ellipsometry for visualization of biomolecular interactions, Anal. Biochem. 232 (1), 69 (1995). DOI: 10.1006/abio.1995.9959.

18. G. Jin, R. Jansson, and H. Arwin, Imaging ellipsometry revisited: Developments for visualization of thin transparent layers on silicon substrates, Rev. Sci. Instrum. 67 (8), 2930 (1996). DOI: 10.1063/1.1147074.

19. G. Jin, Development of biosensor based on imaging ellipsometry, Phys. Stat. Sol. A 205 (4), 810 (2008). DOI: 10.1002/pssa.200777810.

20. Z.-H. Wang et al., A label-free protein microfluidic array for parallel immunoassays, Electrophoresis. 27 (20), 4078 (2006). DOI: 10.1002/elps.200500956.

21. C. Qi et al., Investigation of interaction between two neutralizing monoclonal antibodies and SARS virus using biosensor based on imaging ellipsometry, Biomed. Microdevices 8 (3), 247 (2006). DOI: 10.1007/s10544-006-8305-2. 
22. Y. M. Bae et al., Immunosensor for detection of Legionella pneumophila based on imaging ellipsometry, Mater. Sci. Eng. C 24 (1-2), 61 (2004). DOI: 10.1016/j.msec.2003.09.049.

23. C. Qi et al., Detection of avian influenza virus subtype H5 using a biosensor based on imaging ellipsometry. Biosens. Bioelectro. 25 (6), 1530 (2010). DOI: 10.1016/j.bios.2009.10.030.

24. C. Qi et al., Phage M13KO7 detection with biosensor based on imaging ellipsometry and AFM microscopic confirmation, Virus Res. 140 (1-2), 79 (2009). DOI: 10.1016/j.virusres. 2008.11.010.

25. Z. H. Wang, and G. Jin, A label-free multisensing immunosensor based on imaging ellipsometry, Anal. Chem. 75 (22), 6119 (2003). DOI: 10.1021/ac0347258.

26. Y. Chen, Y. Meng, and G. Jin, Optimization of off-null ellipsometry for air/solid interfaces, Appl. Opt. 46 (35), 8475 (2007). DOI: 10.1364/AO.46.008475.

27. H. Arwin, S. Welin-Klintström, and R. Jansson, Off-null ellipsometry revisited: Basic considerations for measuring surface concentrations at solid/liquid interfaces, $J$ Colloid Interf Sci 156 (2), 377 (1993). DOI: 10.1006/jcis.1993.1125.

28. Z.-H. Wang, and G. Jin, Covalent immobilization of proteins for the biosensor based on imaging ellipsometry, J. Immunol. Methods 285 (2), 237 (2004). DOI: 10.1016/j.jim.2003.12. 002.

29. J. W. A. Findlay et al., Validation of immunoassays for bioanalysis: A pharmaceutical industry perspective, J. Pharm. Biomed. Anal. 21 (6), 1249 (2000). DOI: 10.1016/S07317085(99)00244-7.

30. S. H. Park, J. M. Goo, and C.-H. Jo, Receiver operating characteristic curve practical review for radiologists, Korean J. Radiol. 5 (1), 11 (2004). DOI: 10.3348/kjr.2004.5.1.11.

31. T. Fawcett, An introduction to ROC analysis, Pattern Recogn. Lett. 27 (8), 861 (2006). DOI: 10.1016/j.patrec.2005.10.010.

32. J. Sim, and C. C. Wright, The kappa statistic in reliability studies: Use, iterpretation, and sample size requirements, Phys. Ther. 85 (3), 257 (2005). DOI: 10.1093/ptj/85.3.257. 